\title{
Cephalometric Angular Measurements of the Mandible Using Three-Dimensional Computed Tomography Scans in Koreans
}

\author{
Yong Hyun Kim, Seok Joo Kang, Hook Sun \\ Department of Plastic and Reconstructive Surgery, Busan Paik Hospital, Inje University College of Medicine, Busan, Korea
}

Background We conducted this study to analyze the values of the key cephalometric angular measurements of the mandible using 3-dimensional (3D) computed tomography scans. Methods In the 106 enrolled patients, a 3D cephalometric analysis was performed to measure the angular variables of the mandible. These values were compared between the two sides and between the two sexes.

Results The frontal measurements revealed that the mandibular body curve angle was larger on the left (Lt) side (right [Rt], $141.24 \pm 7.54 ; \mathrm{Lt}, 142.68 \pm 6.94 ; \mathrm{P}=0.002$ ) and the gonial angle was larger on the right side ( $R t, 134.37 \pm 8.44 ; L t, 131.54 \pm 7.14 ; P<0.001)$. The sagittal measurements showed that the gonial angle was larger on the right side (Rt, $134.37 \pm 8.44$; Lt, $131.54 \pm 7.14 ; P>0.05)$. Further, the transverse measurements revealed that the mandibular body curve angle was larger on the right side (Rt, $140.28 \pm 7.05 ; \mathrm{Lt}, 137.56 \pm 6.23 ; \mathrm{P}<0.001)$.

Conclusions These results provide an average of the mandibular angular measurements for the Korean population, establishing a standard for determining surgical patient groups and outcome evaluations in the field of mandible contour surgery.

\section{Keywords Cephalometry / Three-dimensional / Mandible / Angular}

Received: 13 Mar 2015• Revised: 19 Jun $2015 \bullet$ Accepted: 19 Jun 2015

pISSN: 2234-6163 • elSSN: 2234-6171 • http://dx.doi.org/10.5999/aps.2016.43.1.32 • Arch Plast Surg 2016;43:32-37
Correspondence: Hook Sun Department of Plastic and Reconstructive Surgery, Busan Paik Hospital, Inje University College of Medicine, 75 Bokji-ro, Busanjin-gu, Busan 47392, Korea

Tel: +82-51-890-6136

Fax: +82-51-894-7976

E-mail:sun443@naver.com
No potential conflict of interest relevant to this article was reported.

\section{INTRODUCTION}

The chin is one of the major features forming the overall facial profile, and there is an increased demand for jaw bone surgery for both functional and aesthetic purposes [1,2]. Obtaining a preoperative measurement of the anthropometric parameters for craniofacial or orthognathic surgery is important for performing an accurate preoperative assessment, planning surgery, and evaluating the postoperative outcome [3].

In 1922, Pacini [4] first introduced the use of radiographic images for measuring facial anthropometric parameters. Broadbent
[5] further developed this method to create the modern cephalometric analysis in the 1930s. Even recently, conventional cephalometric analysis has been considered an important clinical tool in the planning and evaluation of orthodontics and orthognathic surgery, but it has several well-known limitations such as projective displacement, rotational errors, and linear projective transformation [6].

To overcome these limitations, 3-dimensional (3D) cephalometric analysis using cone-beam computed tomography (СВСТ) in the field of dental and maxillofacial imaging has emerged [7-11]. There is some evidence suggesting that CBCT 
imaging offers better diagnostic potential, leads to better treatment planning, and results in better treatment outcomes than conventional 2-dimensional (2D) imaging [12]. Moreover, 3D cephalometric analysis has better reproducibility than $2 \mathrm{D}$ cephalometry [13]. Thus, it is considered a valuable tool for planning orthognathic surgery.

There have been a few previous reports of 3D cephalometry performed in the Korean population [14,15], and there is virtually no data on $3 \mathrm{D}$ cephalometric analysis of the mandible in the frontal view from the Korean population.

In particular, in the mandible, angular measurement is important in facial contour surgery such as mandible angle reduction, corticectomy, and genioplasty. Interest in such a measurement has increased recently, but there is very little recent data comprising mandibular angular measurements using 3D computed tomography (CT) in Koreans.

Frontal cephalometry has been relatively neglected because of the difficulty in identifying landmarks of the superimposed structures, and because the data obtainable from this view only include information on asymmetries and width of the jaws. However, the use of 3D CT imaging makes it much easier to identify these landmarks, and the additional information may be valuable in cosmetic cases involving the jaw width [13]. This study aims to provide mandibular angular measurements in the sagittal, transverse, and frontal views in Koreans.

\section{METHODS}

\section{Study setting}

A cephalometric analysis of 3D CT imaging was performed in this four-year, single-center, retrospective study. Subjects were selected from the patients who underwent facial CT because of facial trauma between January 2010 and December 2013, on the basis of the following inclusion criteria: (1) Korean adult men or women aged between 18 and 59 years, (2) Patients with no congenital or acquired dentofacial deformities (e.g., cleft lip or palate, craniofacial syndrome, jaw protrusion or retrusion, or post-traumatic deformity), (3) Patients with no obvious facial asymmetry, (4) Patients with no history of oral and maxillofacial surgery, (5) Patients with a Class I occlusal relationship, and (6) Patients with no facial bone fractures.

In the current study, we enrolled 106 Korean individuals $(\mathrm{n}=106)$, including 60 men and 46 women, whose mean age was $32.20 \pm 9.08$ years (range, $20-49$ years).

The current study was approved by the Institutional Review Board (IRB) of our medical institution (IRB approval number: 15-0013).

\section{D CT analysis}

The patients' head was placed in the multi-slice CT (MSCT) scanner (Light speed 16, GE, Milwaukee, Wisconsin, USA) on a foam platform with the Frankfort horizontal plane parallel to the floor. Then, the patients' head was placed in the center of the MSCT scanner by ensuring that the midline light beam coincided with the mid-sagittal plane. The patients were told to bite their teeth in a centric occlusion. The MSCT scans were taken in the extended height mode ( $120 \mathrm{kVp}, 300 \mathrm{Ma}$, Helical mode, field of view: $227.00 \mathrm{~mm}$ ). Then, the 3D model of each head was constructed from the MSCT data using Aquarius iNtuition (TeRarecon, San Mateo, CA, USA). These reconstructed 3D models were used to perform the cephalometric analysis.

\section{Cephalometric landmarks and measurements}

To minimize the identification error in placing the landmarks and obtaining the measurements, the same operator measured them three times at two-week intervals according to their definitions. The detailed definitions of the landmarks used are provided in Table 1. Four angular measurements were obtained using 3D CT imaging for the current study. These measurements were averaged for further statistical analysis. The measurements obtained are presented in Table 2. The Viewbox software (Marosis M-view 5.4, Infinitt Technology, Seoul, Korea) was used to identify conventional cephalometric hard-tissue landmarks and to measure the distance and the angle in each dimension (Figs. 1-3). The data were analyzed to compare between the left and the right sides, and between men and women.

\section{Table 1. Definitions of cephalometric landmarks}

\begin{tabular}{|c|c|}
\hline \multicolumn{2}{|l|}{ Landmarks } \\
\hline B point (B) & $\begin{array}{l}\text { The deepest point between pogonion and the alveolus } \\
\text { of the lower incisors on the midsagittal plane }\end{array}$ \\
\hline Pogonion $(\mathrm{Pg})$ & $\begin{array}{l}\text { The most forward-projecting point on the anterior } \\
\text { margin of symphysis menti on the midsagittal plane }\end{array}$ \\
\hline Gnathion (Gn) & $\begin{array}{l}\text { The most inferior point anterior on the anterior margin } \\
\text { of symphysis menti on the midsagittal plane }\end{array}$ \\
\hline Menton (Me) & $\begin{array}{l}\text { The lowermost point on the symphysis menti on the } \\
\text { midsagittal plane }\end{array}$ \\
\hline $\begin{array}{l}\text { Mandibular body curve } \\
\text { (MBC) }\end{array}$ & $\begin{array}{l}\text { The most convex point on the curvature, midway } \\
\text { between the inner and the outer borders of the } \\
\text { mandibular body }\end{array}$ \\
\hline Gonion (Go) & $\begin{array}{l}\text { The midway point between the lowermost point on the } \\
\text { posterior border of the ramus and the most posterior } \\
\text { point on the lower border of the mandible }\end{array}$ \\
\hline Sigmoid notch (Sig) & The deepest point on the sigmoid notch \\
\hline Condylion (Co) & The uppermost point at the center of the condyle \\
\hline Lateral condyle (Lat Co) & The most lateral point on the mandibular condyle \\
\hline Medial condyle (Med Co) & The most medial point on the mandibular condyle \\
\hline
\end{tabular}


Table 2. Definitions of cephalometric measurements

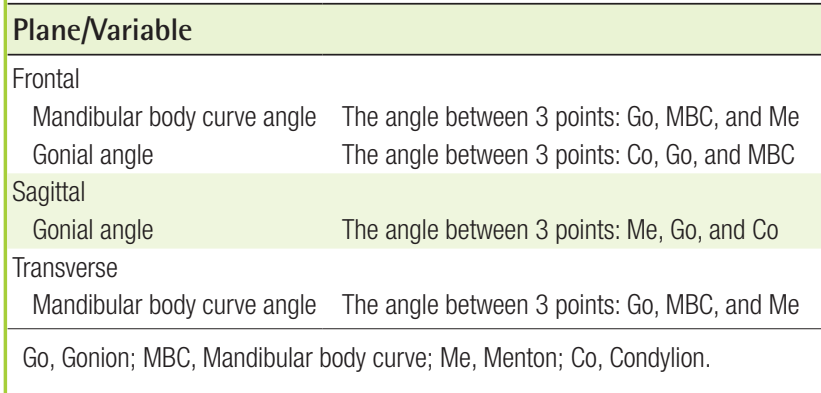

Fig. 1. Schematic representation of frontal landmarks and measurements

FH, Frankfort horizontal plane; Co, condylion; Med Co, medial condyle; Lat Co, lateral condyle; Go, gonion; 1, ramal mediolateral inclination; 2 , condylar width; 3 , condyle to midsagittal plane; 4, gonion to midsagittal plane; 5 , gonial angle; 6 , mandibular body angle.

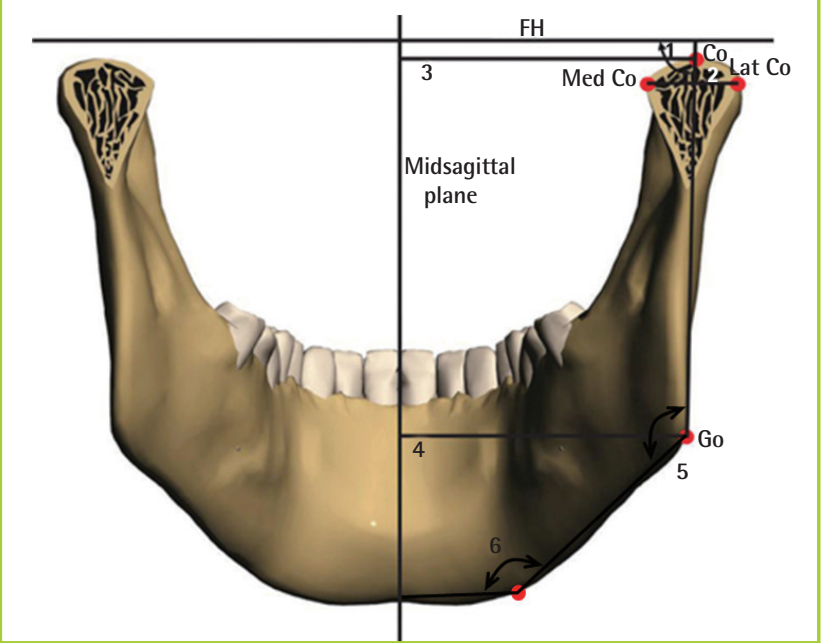

\section{Statistical analysis}

A statistical analysis was carried out using IBM SPSS ver. 21.0 (IBM Corp., Armonk, NY, USA). Linear and angular variables were expressed as mean \pm standard deviation. In addition, a paired $\mathrm{t}$-test was used for comparing the obtained variables between the two sides, and an independent $t$-test was used for comparing the obtained variables between the two sexes.

A P-value of $<0.05$ was considered statistically significant.

\section{RESULTS}

\section{Comparison of measurements between the two sides}

The frontal measurements revealed that both the mandibular body curve angle and the gonial angle showed a statistically significant difference between the right and the left sides $(\mathrm{P}=0.002$ and $\mathrm{P}<0.001$, respectively).

The mandibular body curve angle was larger on the left $(\mathrm{Lt})$
Fig. 2. Schematic representation of sagittal landmarks and measurements

FH, Frankfort horizontal plane; Co, condylion; Go, gonion; Me, menton; 1, condylar height; 2 , condylar anteroposterior inclination; 3 , ramal anteroposterior inclination; 4, ramal length; 5, gonial angle; 6 , mandibular body length.

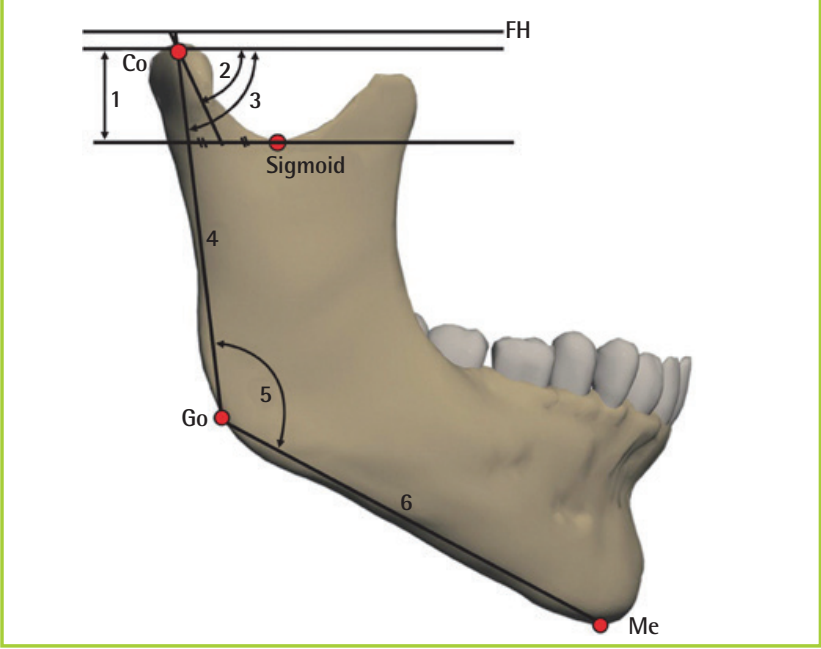

Fig. 3. Schematic representation of transverse landmarks and measurements

Me, menton; MBC, mandibular body curve; Go, gonion; 1, menton angle; 2, mandibular body length; 3, anterior mandibular body length; 4, posterior mandibular body length; 5, MBC angle.

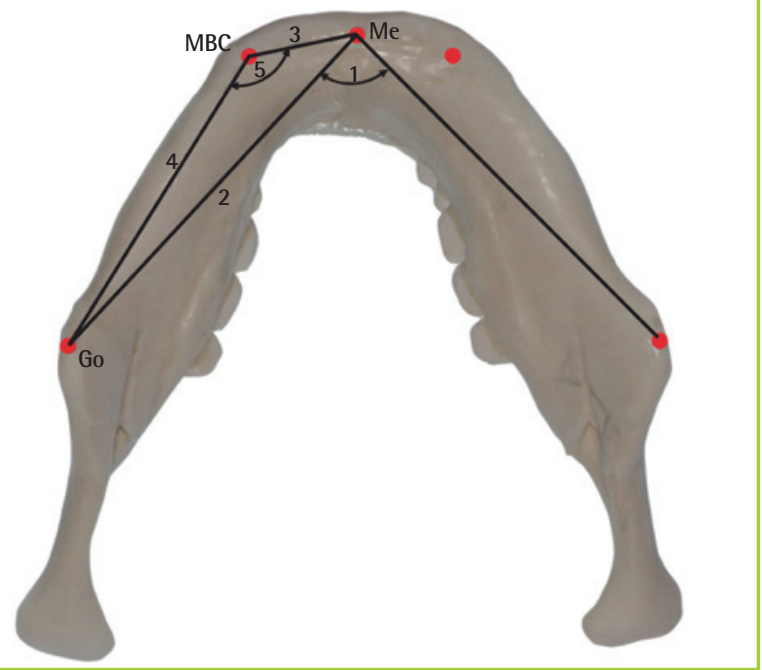

side (right [Rt], 141.24 $\pm 7.54 ; \mathrm{Lt}, 142.68 \pm 6.94 ; \mathrm{P}=0.002$ ), and the gonial angle was larger on the right side (Rt, $134.37 \pm$ 8.44; Lt, 131.54 \pm 7.14; $\mathrm{P}<0.001)$.

The sagittal measurements showed that the gonial angle was larger on the right side without any statistical significance (Rt, 134.37 \pm 8.44 ; Lt, $131.54 \pm 7.14$; $>0.05$ ).

The transverse measurements revealed that the mandibular body curve angle showed a statistically significant difference between the right and left sides $(\mathrm{P}<0.001)$. The mandibular body 


\begin{tabular}{|c|c|c|c|}
\hline Plane/Variable & Right side & Left side & P-value \\
\hline \multicolumn{4}{|l|}{ Frontal } \\
\hline Mandibular body curve angle & $141.24 \pm 7.54$ & $142.68 \pm 6.94$ & $0.002^{\mathrm{a})}$ \\
\hline Gonial angle & $134.37 \pm 8.44$ & $131.54 \pm 7.14$ & $<0.001^{\text {a) }}$ \\
\hline \multicolumn{4}{|l|}{ Sagittal } \\
\hline Gonial angle & $118.68 \pm 14.39$ & $116.21 \pm 8.54$ & Not significant \\
\hline \multicolumn{4}{|l|}{ Transverse } \\
\hline Mandibular body curve angle & $140.28 \pm 7.05$ & $137.56 \pm 6.23$ & $<0.001^{\mathrm{a})}$ \\
\hline
\end{tabular}

curve angle was larger on the right side (Rt, 140.28 \pm 7.05 ; Lt, $137.56 \pm 6.23 ; \mathrm{P}<0.001$ ) (Table 3).

\section{Comparison of measurements between the two sexes}

The frontal measurements revealed that the gonial angle was larger in women, with no statistical significance.

The mandibular body curve angle did not exhibit a statistically significant difference between men and women.

The sagittal measurements showed that women had a larger gonial angle and there was no statistically significant difference between men and women.

The transverse measurements revealed that there was no statistically significant difference in the mandibular body curve angle between men and women (Table 4).

\section{DISCUSSION}

The mandible is a key anatomical structure from both functional and aesthetic perspectives [16]. Patients with mandibular asymmetry or deformity can obtain satisfactory aesthetic outcomes from mandible surgery such as mandible angle reduction, corticectomy, or genioplasty. Considering the 3D configuration and curvature of the mandible and its involvement in dental occlusion, it is mandatory to preoperatively measure the standard cephalometric parameters in these patients [17].

Conventional 2D cephalometric analysis has long been used for measuring key cephalometric landmarks and measurements [18]. The current standard is to use plain lateral and frontal cephalograms for a 2D cephalometric analysis. However, there are well-known limitations to $2 \mathrm{D}$ imaging, such as projective displacement, rotational errors, and linear projective transformation, which interfere with the reliability and reproducibility of these measurements $[6,19]$. Moreover, most 2D measurements are distorted in the presence of facial asymmetry [20]. A 3D cephalometric analysis has been proven to have some benefits over a $2 \mathrm{D}$ analysis, including higher reproducibility $[12,13]$.
Table 4. Comparison of measurements between the two sexes

\begin{tabular}{|c|c|c|c|}
\hline Plane/Variable & $\begin{array}{c}\text { Men } \\
(n=60)\end{array}$ & $\begin{array}{l}\text { Female } \\
(n=46)\end{array}$ & P-value \\
\hline \multicolumn{4}{|l|}{ Frontal } \\
\hline \multicolumn{4}{|c|}{ Mandibular body curve angle } \\
\hline Right & $141.19 \pm 4.60$ & $141.30 \pm 7.55$ & NS \\
\hline Left & $143.35 \pm 6.21$ & $141.82 \pm 7.79$ & NS \\
\hline \multicolumn{4}{|l|}{ Gonial angle } \\
\hline Right & $134.22 \pm 8.47$ & $134.57 \pm 8.48$ & NS \\
\hline Left & $131.00 \pm 7.05$ & $132.25 \pm 7.28$ & NS \\
\hline \multicolumn{4}{|l|}{ Sagittal } \\
\hline \multicolumn{4}{|l|}{ Gonial angle } \\
\hline Right & $118.51 \pm 6.62$ & $118.91 \pm 20.63$ & NS \\
\hline Left & $115.39 \pm 9.53$ & $117.28 \pm 7.02$ & NS \\
\hline \multicolumn{4}{|l|}{ Transverse } \\
\hline \multicolumn{4}{|c|}{ Mandibular body curve angle } \\
\hline Right & $140.17 \pm 7.14$ & $140.42 \pm 7.02$ & NS \\
\hline Left & $137.31 \pm 5.20$ & $137.89 \pm 7.41$ & NS \\
\hline
\end{tabular}

Therefore, we focused on pooling 3D cephalometric measurement data for Korean individuals for further use in craniofacial or orthognathic surgery. We focused on the measurement of the mandibular body curve angles and gonial angles in the frontal view.

Frontal cephalometry has not been routinely performed to make a treatment plan for patients with mandibular deformity. Many structures are superimposed over each other in frontal cephalograms, making it difficult to accurately indicate the landmarks and make measurements. However, this problem can be overcome by using 3D CT images, as the structures are not all superimposed on a film but laid out in a 3D space [21]. The additional information obtained from a frontal cephalometric analysis includes the asymmetry of the mandible and the jaw width, which was considered relatively useless. However, when performing aesthetic surgery, this information is valuable for achieving jaw symmetry [13]. In particular, frontal cephalometry is essential for patients with square jaws who are scheduled to undergo mandibular chin-body ostectomy and corticectomy. There have been reports of a cephalometric analysis of the mandible length and the gonial angle in the lateral view of $2 \mathrm{D} C \mathrm{CT}$ [22]. However, in the lateral view, it is difficult to evaluate the degree of harmony between the craniofacial structures on the basis of the width and the length of the face, and the frontal view is valuable in this regard [23].

Compared to the many previous studies using $\mathrm{CBCT}$ to acquire 3D imaging for a cephalometric analysis, MSCT was used in this study. Although the advantage of CBCT may be its availability for use by a dentist or an orthodontist without the need 
for a radiologist, the images acquired from MSCT are more precise with less distortion [24]. It is perfectly capable of obtaining high-quality images for a cephalometric analysis; however, it has one disadvantage: it exposes the patient to more radiation than CBCT [21].

From the statistical analyses, in the Korean population, the gonial angle was statistically significantly larger on the right side in the frontal view, and the gonial angle was larger on the right side in the sagittal view, although this was not statistically significant. This indicates that the right gonial angle is larger in Koreans, with asymmetry present in general. Therefore, for plastic and orthodontic surgery, surgeons should consider the asymmetry of both sides in order to control symmetry.

In the gender-based comparisons, no statistically significant difference was observed, but the gonial angle was larger in women in both the frontal view and the sagittal view, which contradicts the fact that the mandibular bony structure is generally more angulated in men than in women. More studies are necessary to investigate this relationship in the future.

As this is one of the early studies on the accumulation of 3D cephalometric measurement norms, further studies on the topic are necessary. It is important to analyze the relationship between bony structures and the soft tissue, as the shape of the underlying bony structure and how the overlying soft tissue is formed may differ between individuals. Moreover, further study is needed to compare the cephalometric measurements between Koreans and other ethnic populations.

In summary, we performed a 3D cephalometric analysis of the angular measurements of the mandible in Korean individuals.

The significance of this study lies in presenting an average of the mandibular angular measurement in Koreans using 3D CT of the mandibular angles, which are difficult to measure in $2 \mathrm{D}$ and are needed to develop a standard for determining surgical patient groups and outcome evaluations in mandible contour surgery in the future.

\section{REFERENCES}

1. Jegal JJ, Kang SJ, Kim JW, et al. The utility of a three-dimensional approach with T-shaped osteotomy in osseous genioplasty. Arch Plast Surg 2013;40:433-9.

2. Wang Y, Kotsis SV, Chung KC. Applying the concepts of innovation strategies to plastic surgery. Plast Reconstr Surg 2013;132:483-90.

3. Szychta P, Rykala J, Kruk-Jeromin J. Individual and ethnic aspects of preoperative planning for posttraumatic rhinoplasty. Eur J Plast Surg 2011;34:245-9.

4. Pacini SJ. Roentgen ray anthropometry of the skull. J Ra- diogr 1922;3:230-8.

5. Broadbent BH. A new $\mathrm{x}$-ray technique and its application to orthodontia. Angle Orthod 1931;1:45-66.

6. Kapila S, Conley RS, Harrell WE Jr. The current status of cone beam computed tomography imaging in orthodontics. Dentomaxillofac Radiol 2011;40:24-34.

7. $\mathrm{Oz} \mathrm{U}$, Orhan $\mathrm{K}, \mathrm{Abe} \mathrm{N}$. Comparison of linear and angular measurements using two-dimensional conventional methods and three-dimensional cone beam CT images reconstructed from a volumetric rendering program in vivo. Dentomaxillofac Radiol 2011;40:492-500.

8. Liedke GS, Delamare EL, Vizzotto MB, et al. Comparative study between conventional and cone beam CT-synthesized half and total skull cephalograms. Dentomaxillofac Radiol 2012;41:136-42.

9. Cevidanes LH, Styner MA, Proffit WR. Image analysis and superimposition of 3-dimensional cone-beam computed tomography models. Am J Orthod Dentofacial Orthop 2006; 129:611-8.

10. Lin HH, Chuang YF, Weng JL, et al. Comparative validity and reproducibility study of various landmark-oriented reference planes in 3-dimensional computed tomographic analysis for patients receiving orthognathic surgery. PLoS One 2015; 10:e0117604.

11. van Vlijmen OJ, Maal T, Berge SJ, et al. A comparison between 2D and 3D cephalometry on CBCT scans of human skulls. Int J Oral Maxillofac Surg 2010;39:156-60.

12. van Vlijmen OJ, Kuijpers MA, Berge SJ, et al. Evidence supporting the use of cone-beam computed tomography in orthodontics. J Am Dent Assoc 2012;143:241-52.

13. Farronato G, Garagiola U, Dominici A, et al. “Ten-point” 3D cephalometric analysis using low-dosage cone beam computed tomography. Prog Orthod 2010;11:2-12.

14. Lee SH, Kil TJ, Park KR, et al. Three-dimensional architectural and structural analysis: a transition in concept and design from Delaire's cephalometric analysis. Int J Oral Maxillofac Surg 2014;43:1154-60.

15. Bayome M, Park JH, Kook YA. New three-dimensional cephalometric analyses among adults with a skeletal Class I pattern and normal occlusion. Korean J Orthod 2013;43: 62-73.

16. Hofer SO, Payne CE. Functional and Aesthetic Outcome Enhancement of Head and Neck Reconstruction through Secondary Procedures. Semin Plast Surg 2010;24:309-18.

17. Schaaf H, Malik CY, Howaldt HP, et al. Evolution of photography in maxillofacial surgery: from analog to $3 \mathrm{D}$ photography - an overview. Clin Cosmet Investig Dent 2009;1: 39-45. 
18. Shahidi S, Oshagh M, Gozin F, et al. Accuracy of computerized automatic identification of cephalometric landmarks by a designed software. Dentomaxillofac Radiol 2013;42: 2011 0187.

19. Lin HS, Li JD, Chen YJ, et al. Comparison of measurements of mandible growth using cone beam computed tomography and its synthesized cephalograms. Biomed Eng Online 2014;13:133.

20. Xia JJ, Gateno J, Teichgraeber JF. New clinical protocol to evaluate craniomaxillofacial deformity and plan surgical correction. J Oral Maxillofac Surg 2009;67:2093-106.

21. van Vlijmen OJ, Maal TJ, Berge SJ, et al. A comparison between two-dimensional and three-dimensional cephalometry on frontal radiographs and on cone beam computed to- mography scans of human skulls. Eur J Oral Sci 2009;117: 300-5.

22. Oh S, Kim CY, Hong J. A comparative study between data obtained from conventional lateral cephalometry and reconstructed three-dimensional computed tomography images. J Korean Assoc Oral Maxillofac Surg 2014;40:123-9.

23. Ferrario VF, Sforza C, Miani A, et al. Craniofacial morphometry by photographic evaluations. Am J Orthod Dentofacial Orthop 1993;103:327-37.

24. Medelnik J, Hertrich K, Steinhauser-Andresen S, et al. Accuracy of anatomical landmark identification using different CBCT- and MSCT-based 3D images: an in vitro study. J Orofac Orthop 2011;72:261-78. 


\section{Discussion}

\section{Cephalometric Angular Measurements of the Mandible Using Three-Dimensional Computed Tomography Scans in Koreans}

Kang Young Choi ${ }^{1}$, Nak Heon Kang ${ }^{2}$

${ }^{1}$ Department of Plastic and Reconstructive Surgery, Kyungpook National University School of Medicine, Daegu; ${ }^{2}$ Department of Plastic and Reconstructive Surgery, Chungnam National University School of Medicine, Daejeon, Korea

Radiologic imaging has become an essential instrument in medicine. In computed tomography (CT) imaging, the X-ray emitter and sensors rotate about the axial plane and overlapping X-ray beams penetrate the body from various directions. Most internal organs differ from each other in radiation-absorptive density, and this leads to variations in the amount of radiation detected by the sensor. Computers analyze this variability in radiation absorption and tomographically deduce two-dimensional (2D) data on the internal tissue density and shape. To sum up, CT imaging produces computer images after measuring $\mathrm{X}$-rays from various directions and processing their radiation-absorptive density.

With recent advances in image processing technology, raw data from CT machines can be used to construct three-dimensional (3D) tomographic data, known as "volume rendering." Unlike surface rendering, 3D images from volume rendering allow rotation and translation along all three axes ( $\mathrm{x}, \mathrm{y}$, and $\mathrm{z})$, as well as observation of stair-step artifacts and the inner surface of the bone cortex. This enables simple and rapid interpretation of the internal anatomy and physiology [1]. Recently, even $3 \mathrm{D}$ printing technology using 3D CT has been evolving into patient-specific individualized medicine [2].

In CT images, distances are calculated using the number of pixels between two points. This pixel-distance is converted to the physical distance of the object measured using a pixel-space to millimeter conversion factor (i.e., Pixel Spacing) stored in the Digital Imaging and Communications in Medicine (DICOM) Tag $(0028,0030)$ (Fig. 1). Using the 'image calculate' parameter, distances measured on 2D CT images correlate extremely well with the physical objects the images represent. All of this can readily be accomplished on picture archiving and communication system (PACS) monitors. However, distance projection in a $3 \mathrm{D}$ model is a different matter. The images are simplified

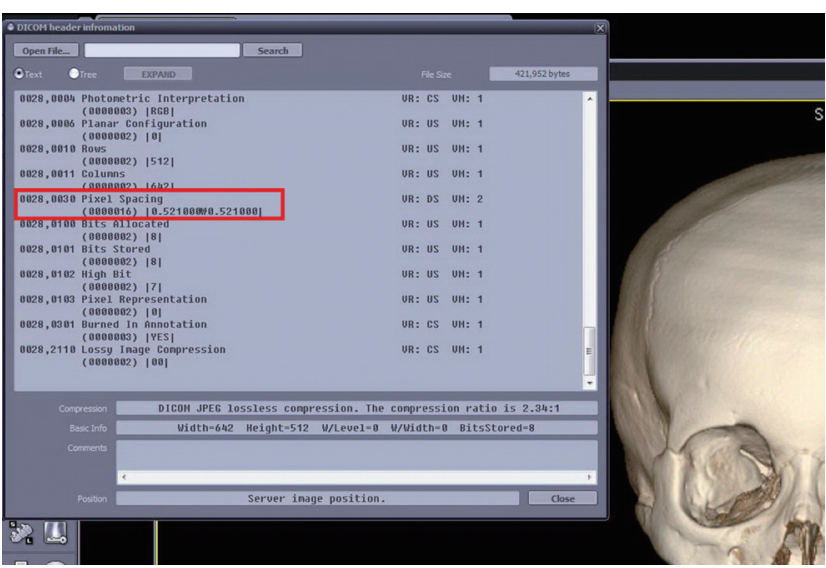

Fig. 1. Digital Imaging and Communications in Medicine (DICOM) Tag Information. The red box indicates a DICOM Tag (0028, 0030 Pixel Spacing), and other information stored with this DICOM Tag.

2D projections of the real 3D model, which is transmitted from the PACS server to the client without the metadata such as Pixel Spacing, monitor resolution, image magnification, or z-axis values. Thus, the information needed to translate pixel distance into physical distance is lost during this process, which implies that interpersonal anthropometric comparisons are not possible with 3D CT images. Of course, 3D images can still allow for intrapersonal comparisons (e.g., before and after an intervention), but this can only represent relative, not absolute, changes in anthropometry [3].

In the study titled "Cephalometric parameters of the mandible on three-dimensional computed tomography scans in Koreans," the authors sought to establish linear cephalometric means for the Korean population using 3D CT reconstructions. Unlike 2D cephalometry, conventional 3D CT allows for absolute measurement of cephalometric angles. However, absolute linear measurements cannot be obtained unless imaging and capturing settings are the same in every object. This is why there is no published research on absolute measurements using $3 \mathrm{D}$ CT despite numerous potential applications for such information.

In a study on the effect of aging on the midmaxilla, Shaw and Kahn [4] had used 3D CT images for measuring the various angular parameters. In a similar study using 3D CT to evaluate mandibular aging, Pessa et al. [5] had used sectional imagesnot 3D models - for direct measurement of the mandible. In addition, Oh et al. [6] argued that only angular measurements would be statistically significant in a study comparing 3D CT images to conventional lateral cephalometry. 

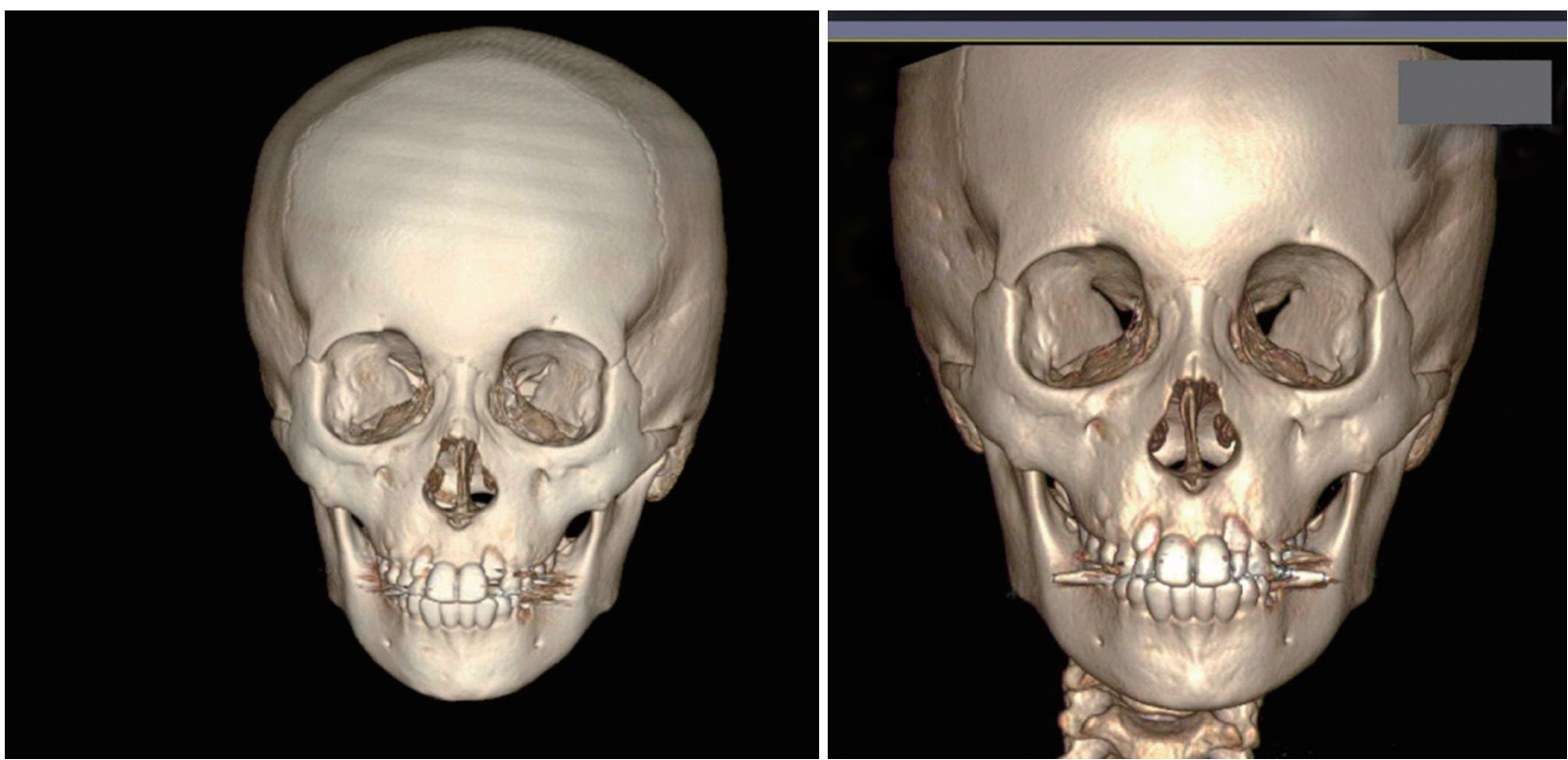

Fig. 2. Three-dimensional-computed tomography (3D CT) reconstructed model. It is difficult to use one 3D CT reconstructed image as a reference for another due to the different absolute values generated by differing conditions such as display field of view, tilt, and window, as in the two images shown here.

In reality, absolute values differ even for a single patient whose CT scans were obtained under differing conditions (Fig. 2). However, if the various conditions of CT scans are unified, not only the measurement of the absolute values but also interpersonal comparison is possible. The following factors are needed: First, the physical values for the CT scans must be the same across all scans. These include the field of view, angle value, $\mathrm{z}$ axis value, monitor resolution, and image magnification. With these conditions held constant, images can be reconstructed in the display window on the screen and measurements can be screen-captured and sent to the PACS. In such contexts, the linear and angular measurements are representative of the physical values.

To conclude, retrospective studies on 3D CT anthropometry can be difficult because of confounding factors that can influence comparisons. Therefore, to obtain accurate data, investigators should design studies prospectively with the variables of the CT scanner set consistently for all images obtained. Even under such conditions, limitations still exist in volume measurement and comparisons in individual physical properties (i.e., Hounsfield units).

\section{REFERENCES}

1. Kuszyk BS, Heath DG, Bliss DF, et al. Skeletal 3-D CT: advantages of volume rendering over surface rendering. Skeletal Radiol 1996;25:207-14.
2. Choi JW, Kim N. Clinical application of three-dimensional printing technology in craniofacial plastic surgery. Arch Plast Surg 2015;42:267-77.

3. Whyms BJ, Vorperian HK, Gentry LR, et al. The effect of computed tomographic scanner parameters and 3-dimensional volume rendering techniques on the accuracy of linear, angular, and volumetric measurements of the mandible. Oral Surg Oral Med Oral Pathol Oral Radiol 2013;115:68291.

4. Shaw RB Jr, Kahn DM. Aging of the midface bony elements: a three-dimensional computed tomographic study. Plast Reconstr Surg 2007;119:675-81.

5. Pessa JE, Slice DE, Hanz KR, et al. Aging and the shape of the mandible. Plast Reconstr Surg 2008;121:196-200.

6. Oh S, Kim CY, Hong J. A comparative study between data obtained from conventional lateral cephalometry and reconstructed three-dimensional computed tomography images. J Korean Assoc Oral Maxillofac Surg 2014;40:123-9.

Correspondence: Nak Heon Kang

Department of Plastic and Reconstructive Surgery, Chungnam National University School of Medicine, 282 Munhwa-ro, Jung-gu, Daejeon 35015, Korea

Tel: +82-42-280-7380, Fax: +82-42-280-7384, E-mail: nhk488@yahoo.com

No potential conflict of interest relevant to this article was reported.

Received: 5 Dec 2015 • Revised: 28 Dec 2015 • Accepted: 28 Dec 2015 pISSN: 2234-6163 • elSSN: 2234-6171

http://dx.doi.org/10.5999/aps.2016.43.1.38 • Arch Plast Surg 2016;43:38-39 